\title{
TOMOGRAFÍA: MÁS ALLÁ \\ DE LAS APLICACIONES MÉDICAS
}

\section{Franklin Gavilánez}

\begin{abstract}
Tomography using CT scans and MRI scans is now well-known as a medical diagnostic tool which allows for detection of tumors and other abnormalities in a noninvasive way, providing very detailed images of the inside of the body using low dosage $\mathrm{X}$-rays and magnetic fields. They have both also been used for determination of material defects in moderate size objects. In medical and other applications they complement conventional tomography. There are many situations where one wants to monitor the electrical conductivity of different portions of an object, for instance, to find out whether a metal object, possibly large, has invisible cracks. This kind of tomography, usually called Electrical Impedance Tomography or EIT, has also medical applications like monitoring of blood flow. While CT and MRI are related to Euclidean geometry, EIT is closely related to hyperbolic geometry. A question that has arisen in
\end{abstract}

the recent past is whether there is similar "topographic" method to monitor the "health" of networks. Our objective is to explain how EIT ideas can in fact effectively be used in this context.

\section{Introducción}

Problemas tomográficos aparecen en varios contextos, desde el monitoreo de redes hasta el procesamiento de señales, así como situaciones relacionadas a biología y medicina, consecuentemente, hay diferentes tipos de aplicaciones de tomografía y tópicos misceláneos en análisis harmónico y complejo que se relacionan. Estos problemas son vistos como problemas inversos y entre estos aquellos de índole discreto sobre conductividad en redes, lo cual se basa en ideas y métodos tomográficos ya existentes para el caso continuo. Mi investigación se enmarca en este tipo de problemas que realizo con la colaboración de Carlos Berenstein, quien

* Exalumno del Colegio Salesiano "Santo Tomás Apóstol" de Riobamba-Ecuador. Profesor de Matemática en Montgomery College, Rockville, Maryland-USA. 
es un experto mundial en el tema e investigador en la Universidad de Maryland en College Park, Estados Unidos.

El impacto causado por actividades investigativas de Berenstein es grande. Parte del trabajo de Él es motivado por algunas interrogantes que aparecen en el estudio de procesamiento de señales e imágenes (obtenidas con uso de rayos X). Para ser conciso, esta investigación se origina en la pregunta de cómo obtener la máxima cantidad de resolución de la imagen de algún órgano humano, por ejemplo, minimizando la cantidad de radiación o tiempo de exposición de acuerdo al contexto, precisamente esto llevó a Berenstein a crear una nueva configuración de scanners de tomografía computarizada que permite la localización, es decir, la obtención de información y procesamiento de imágenes sólo de la región de interés, que es una característica que los scanners convencionales no pueden lograr. Por esto, Berensteín recibió una patente del gobierno de los Estados Unidos (patente US-5953388). Uno infiere que además de las aplicaciones en radiología médica como por ejemplo mejorar la resolución en el área de interés clínico sin necesidad de incrementar la exposición total de la fuente de energía, la real motivación fue facilitar el escaneo de objetos grandes tales como partes de cohetes o alas de aviones para determinar la existencia de resquebrajamientos. Una variación de ésta es considerada, en el problema de conductividad inversa así como el problema de la detección de roturas en materiales. Ahora describiré como esta situación es análoga a preguntas naturales acerca de la performance y seguridad de redes y que permite la detección temprana de ataques a ellas.

La combinación de ideas tomográficas y la matemática, específicamente deconvolución, está siendo usado en nuevos equipos de radiología desarrollado por C. Jeanguillaume, un radiólogo en Angers (Francia). Esta misma combinación de ideas tomográficas es también fundamental para resolver el problema de cómo obtener rápida y robusta discriminación de diferentes fuentes de sonido con un mínimo número de micrófonos (identificación o separación de sonidos). Esto describe brevemente la enorme influencia de ideas tomográficas y su importancia.

Mi investigación se centra en tomografía, transformada de Radón, problemas inversos en redes (grafos particulares) y aplicaciones. En nuestra sociedad, la transformada de Radón Euclidiana juega un rol fundamental en la obtención de imágenes 
médicas. Por ejemplo, en el plano Euclideo, tenemos lo que conocemos como tomografía de rayos $X$. En el caso del plano hiperbólico (no Euclideo), la transformada de Radón hiperbólica aparece naturalmente al estudiar el problema de conductividad inverso también conocido como tomografía de impedancia eléctrica. EIT (por sus siglas en inglés), o problema de Neumann-Dirichlet. Existe también trabajo numérico para detectar roturas en un plato metálico usando mediciones solamente en la frontera del plato. Sería interesante poder usar ondeletas en el contexto de la geometría hiperbólica para estudiar la transformada de Radón. Por otro lado, hay evidencia generada por computadores que la transformada de Radón hiperbólica en el plano puede ser útil para entender redes, por ejemplo, redes de comunicación. En el caso de redes, si uno quiere obtener información acerca del interior de la red o estimar parámetros a lo largo de conexiones internas, suena natural considerar ideas análogas directamente en el contexto de grafos, lo que uno podría llamar tomografía de redes, es decir, entender los modelos de tráfico en internet y garantizar su seguridad. Al menos dos de estos problemas tienen una interpretación natural en términos de EIT:
(1) Mediante observaciones de características de tráfico de un punto a otro punto, reconstruir las características de la red.

(2) Desarrollar métodos para detectar el comienzo de una interrupción de la red.

Desde el punto de vista de tomografía de redes. Uno puede referirse al trabajo de Fan Chung, Graham y sus colaboradores para determinar la estructura de un grafo usando un enfoque probabilístico al problema de Neumann Dirichlet, lo que ellos llaman "firing chips" sobre grafos muy perceptivo. En términos no técnicos, si un nodo o un grupo de nodos ha parado de funcionar entonces la estructura del grafo ha cambiado. Por otro lado, parte de la red (ejemplo una red de cajeros automáticos o una autopista) podría volverse tan saturada por el tráfico que se vuelve prácticamente infuncional aunque la estructura del grafo (red) no haya cambiado. En este caso, uno quisiera mantener información de la cantidad de "tráfico" entre los nodos, esto es, considerar un grafo con pesos, para detectar el comienzo de tal interrupción a través de la transformación de Neumann-Dirichlet. Esto puede ser fácilmente considerado como un problema de Neumann Dirichlet del operador de Laplace que 
involucre el tráfico presente, es decir debemos considerar en el grafo un Laplaciano con pesos.

Otros problemas tales como problemas que involucran la identificación de grafos han estado entre los más importantes y famosos problemas abiertos en teoría de grafos.

La mayoría del trabajo en esta dirección se ha concentrado en teoría espectral de grafos, sobre la realización de grafos con distancias dadas y sobre la reconstrucción de grafos a partir de subgrafos sin vértices. Por lo tanto, la teoría espectral ha sido una de las más significantes herramientas usadas para estudiar grafos y ha conllevado al notable progreso en el estudio acerca de los problemas mencionados anteriormente. Pero, como es bien sabido, grafos no son en general completamente caracterizados por su espectro y esto motiva mi investigación en una forma más concisa que la expongo aquí:

\section{Problemas}

Cuantificar la correspondencia entre EIT, tomografía en el caso continuo y el modelo discreto sobre grafos (redes).

Extender al caso discreto las técnicas ya usadas en el caso continuo de tomografía.

\section{Objetivo}

Descubrir rápidamente si la red ha sido comprometida y en donde.

Desarrollar un modelo matemático del Internet que nos permita determinar rápidamente donde y cuando interrupciones de la red ocurren. Los métodos a desarrollarse son basados en ideas tomográficas.

\section{Aplicaciones}

Permitirá la determinación temprana de la ubicación de los ataques a una red por lo tanto permitiendo medidas rápidas para detener $\mathrm{o}$ al menos contener el ataque.

Determinar y parar la intrusión en redes ad hoc grandes como la internet, redes móviles, redes de pague por ver, etc.

\section{Metodología}

Extensión de los métodos tomográficos ya bien desarrollados en el caso de medios continuos para determinar distribuciones de densidad en una forma no invasiva. La idea es similar a aquellas usadas en tomografía médica y en la determinación de defectos en materiales.

Redes, en particular, redes de comunicación como la internet se han vuelto una parte esencial del diario vi- 
vir y por ello las interrupciones pueden causar consecuencias muy serias $y$, por lo tanto, aparece la necesidad de prevenir o al menos detectar tempranamente tales interrupciones. El problema a enfrentar es aquel de obtener información de la estructura interna de la red a partir de la recolección de datos en la periferia de la red. Esto es análogo a problemas tomográficos que han sido estudiados en el caso continuo y es precisamente esta analogía mi fuente de Investigación.

El problema de descubrir la estructura interna de la red en forma detallada a partir de la recolección de dalos en la frontera de la red puede ser visto como un tipo de problema inverso análogo a aquellos que existen en tomografía convencional sólo que en este caso estamos en un ambiente discreto. La situación más realista corresponde a usar colecciones más pequeñas de datos para obtener información solamente de una subregión de la red lo cual corresponde a la tomografía localizada en el caso convencional. Una de las formas de tratar de entender lo que está ocurriendo es visualizar el grafo dirigido que representa a la red en el espacio hiperbólico de dimensión 3 $\mathrm{o}$ inclusive en el espacio hiperbólico de dimensión 2, dado que en estos espacios el volumen de la esfera crece exponencialmente en función del radio, lo cual es distinto al caso de la esfera en el espacio Euclideo de dimensión 3 , respectivamente de dimensión 2 .

Nosotros hemos desarrollado una innovadora formulación matemática de estos problemas, usando esta representación de la red incrustada en el plano hiperbólico. En esta representación, los caminos entre nodos se vuelven las geodésicas del plano hiperbólico. Luego, nuestra formulación y métodos de solución al problema confirman que la correcta tomografía a usar no es la Euclidiana sino aquella del espacio hiperbólico real en dos dimensiones o tres. Hay varias razones por las que este modelo parece ser el correcto. Primero, la evidencia empírica y recientes análisis de la topología de la internet y conexiones de redes parecidas a la internet, las cuales han mostrado que el número de nodos de las redes tienen una ley de crecimiento polinomial con respecto a cualquier punto de la red. Segundo, el trabajo de visualización de Munzer (y varios reportes de CAIDA) que muestra como estos tipos de redes pueden ser visualizados en el espacio hiperbólico real.

El principal objetivo de mi investigación es obtener algoritmos computacionalmente eficientes para solucionar tales problemas inversos, es 
decir, el procedimiento para determinar el perfil interno de la red. Mi idea se basa en trabajos previos de Berenstein donde Él estudia un problema inverso clásico de ecuaciones diferenciales parciales, el problema de conductividad inversa o tomografía de impedancia eléctrica, EIT mencionado anteriormente. Este problema es el análogo en el caso continuo de problemas de tomografía en redes eléctricas.

Los problemas de EIT que aparecen de problemas tomográficos son más similares a problemas inversos en redes eléctricas discretas como los investigados y resueltos por Curtis y Morrow [5] en el caso de una red cuadrada de resistencias (lattice). Nuestra aproximación combina los métodos de Curtís y Morrow con los antiguos métodos tomográfios para árboles y grafos, al mismo tiempo que extiende estos métodos a modelos probabilísticos y situaciones como el caso de arbitrarios grafos finitos donde algún tipo de peso o trafico w se ha definido.

Curtís y Morrow muestran que la conductividad $\omega$ puede ser determinada en forma única y proponen un algoritmo para computar $\omega$. En el caso de grafos arbitrarios planos y finitos en los que se definen un peso $\omega$, junto con Berenstein y S-Y Chung se probó un resultado de unicidad el cual permite determinar el peso a partir de valores conocidos en la frontera, y que lo describo a continuación: dados dos pesos positivos, $\omega_{1} y \omega_{2}$ entonces $\omega_{1}=\omega_{2}$ si y solamente si el mapeo de Neumann a Dirichlet asociado a $\omega_{1}$ es igual al mapeo de Neumann a Dirichlet asociado a $\omega_{2}$.

En mi investigación trato de encontrar métodos computacionales que sean efectivos para monitorear subconjuntos específicos $S$ de grafos planos arbitrarios (regiones de interés) provistos de un peso a partir de la fundón input-output correspondiente a caminos que han cruzado tales regiones y desde aquí determinar, por ejemplo, áreas congestionadas, o mejor aún, anticipar áreas que se congestionarán y así poder recomendar medidas para evitar la paralización del trafico (peso).

Conseguido esto, mi siguiente objetivo es desarrollar una estrategia para determinar el peso $w$ para el caso de grafos en general provistos de un peso. Para poder lograr esto, considero regiones de interés $S$ relativamente pequeñas y con elecciones apropiadas de datos $\delta$ del problema de Neumann asociado al peso positivo $\omega$ para obtener soluciones únicas $U_{\delta}$ las cuales satisfacen $\Delta_{\omega} U_{\delta} \equiv 0$ en $S$ que es la ecuación de Laplace correspondiente al Laplaciano con peso $\Delta_{\omega}$, (es decir $U_{\delta}$ es $\omega$ - harmónica) $y$ 
también la condición $\frac{\partial \mathrm{U}_{\delta}}{\partial \mathrm{n}_{\omega}} \equiv 0$ en la frontera de $S$, indicada por $\partial S$, la derivada normal asociada al peso $\omega$. Dado que $S$ es un grafo relativamente simple, $U_{\delta}$ es obtenida a partir de apropiadas elecciones de valores de $\delta$ $y$ de aquí $\omega$ se encuentra numéricamente utilizando algún software existente para resolver sistemas de algebra lineal. Esto permitirá determinar una estrategia para encontrar $\omega$ para el caso de grafos generales $S$, donde la matriz $T$, asociado al sistema linear que tiene w, como incógnita es una matriz muy grande pero sin muchos ceros.

Me parece que tal estrategia se basará en ondeletas para el caso discreto, es decir, considerando apropiadas funciones $\Delta$, que se comportan como ondeletas y que serán definidas en la frontera de $S$, para producir una matriz $T$, con muchos ceros.

En el caso de problemas inversos continuos sobre un conjunto, uno observa que la condición de desvanecimiento de una ondeleta corresponde exactamente al hecho que cualquier solución del problema continuo de Neumann tiene promedio cero en todo el conjunto y esto es debido al teorema de Green. Antes de retornar al problema que nos ocupa, quiero indicar otra característica del resultado principal en [4], el cual pienso que puede ser útil cuando se estudian redes muy grandes. Es el llamado principio de localización. En el caso continuo, considerando la transformada de Radón, esto significa lo siguiente: Supongamos que uno está solamente interesado en determinar los valores de una función $f$ en una subregión $S_{1}$ de una entera región So y que esto se logra usando rayos $X$, entonces, solamente se necesita que los rayos $X$ pasen a través de $S_{1}$ para determinar los valores de $\mathrm{f}$ en $S_{1}$. Para ser más preciso, uno necesita que los rayos $X$ pasen a través de un conjunto $S_{1}{ }_{1}$ ligeramente más grande que $S_{1}$. El ingrediente clave para probar este resultado fue el uso de ondeletas, principalmente el hecho que las ondeletas son funciones con promedio cero, esto es que el integral sobre la frontera es igual a cero.

Regresando al problema sobre redes, estamos al momento trabajando en desarrollar una teoría análoga a aquella de las ondeletas para implementar explícitamente la determinación del peso o las cargas del tráfico a lo largo de las aristas de la red. Debo indicar que para redes muy grandes el resultado de localización mencionado antes puede ser muy importante dado que nos permitiría monitorear solamente las regiones de interés dentro de la red en forma muy eficiente.

El ambiente matemático que se define para estudiar el problema 
mencionado antes es aquel de grafos con un peso $\omega$ definido en sus aristas. A parte de esto, es asumido que el subyacente grafo es conocido por quienes están monitoreando la red. En este contexto discreto yo modelo el problema en la siguiente forma. Consideremos un grafo finito conectado $G=G(E, V)$, donde $E$ denota el conjunto de aristas de $G y V$ el conjunto de nodos. Dos nodos $x$ y $y$ son adyacentes y se indica por $x \sim y$, si ellos son los extremos de una arista en $G$. Es asumido también que hay un subconjunto no vacío muy específico $\partial G$ de $G$, llamado la frontera de $\mathrm{G}$ la cual representa los nodos que son accesibles a nosotros para monitorear el tráfico (peso). Adicionalmente, se asume que a cada arista en $E$ se asocia un número no negativo $\omega(x, y)$ el cual corresponde al tráfico entre los extremos $x$ y $y$ de la arista. El grado $\delta_{\omega} x$ de un nodo $x$ en el grafo $G$ con peso $\omega$ es definido por

$$
\delta_{\omega} x=\Sigma_{y \in v} \omega(x, y)
$$

El operador Laplaciano correspondiente a este peso $\omega$ se define como $\Delta_{\omega} f(x)=\Sigma_{y \in v}[f(y)-f(x)] . \frac{\omega(x, y)}{\delta_{\omega} x}, x \in V$ donde f es una función que actúa sobre los vértices.
En este modelo, hay dos tipos de interrupciones de tráfico de datos que podrían presentarse. En uno de ellos, las interrupciones ocurren cuando una arista "deja de existir", en este caso la "topología" del grafo ha cambiado. Este tipo de interrupción esta fuera del objetivo de mi investigación. En el otro, los pesos cambian debido a que el tráfico aumenta, es decir, la configuración de la red permanece igual pero los pesos han ya sea aumentado o permanecido igual.

Uno concluye que escogiendo una base como el conjunto de datos del problema de Neumann uno puede decidir si hay un incremento de tráfico o no en algún lugar de la red. Aunque este es solamente un teorema de unicidad, sin embargo, este conlleva a la posibilidad de computar efectivamente el peso actual a partir del conocimiento de los datos del problema de Dirithlet (output) por convenientes elecciones de los datos del problema de Neumann (input). Esto a su vez conlleva a la pregunta natural sobre la determinación efectiva del peso $\mathrm{w}$ en dicho grafo.

De nuevo, monitorear la red en este contexto matemático significa que estamos monitoreando la cantidad de trafico w (cargas o pesos) a lo largo de las aristas durante un período en el cual la red está trabajando y lo que 
queremos es tomar algunas medidas preventivas en caso de que el tráfico a lo largo de algunas aristas esta cerca de sobrecargar aquellas aristas y causar una interrupción mayor. Nótese que en este modelo si una arista se vuelve saturada, es prácticamente lo mismo que decir que la arista no existe más y, por lo tanto, el grafo ha cambiado. Por supuesto, el punto es luego tener me- didas a mano para solucionar este problema. Antes de finalizar nótese que uso el termino tomografía de redes en realidad para indicar que estoy pensando en este problema en términos de una analogía muy cercana al uso de la transformada de Radón en un número de aplicaciones en el caso continuo, por ejemplo, aquel de los CT escáner.

\section{Bibliografía}

BERENSTEIN, C. A., and CASADIO TARABUSI, E.C.

1994 The inverse conductivity problem and the hyperbolic Randon transform, "75 years of Radon Transform". S. Gindikin and P. Michor, editors. International Press.

BERENSTEIN, C. A., and E. C. CASADIO TARABUSI

1996 Integral geometry in hiyperbolic spaces and electrical impedance tomography. SIAM J. Appl. Math. 56, 755-764.

BERENSTEIN, C. A., and Chung, S-Y

$2005 \omega$-Harmonic functions and inverse conductivity problems on networks, SIAM J. Appl. Math. 65, No. 4, 1200-1226.

BERENSTEIN, C. A., WALNUT, D., RASHID-FARROKHI, F., and RAY LIU, K. J.

1997 Wavelet-based multiresolution Iocal tomography. IEEE. Transactions on Image Processing. Vol, 06, no. 10.pp. 1412-I430.

BERENSTEIN, A. et al.,

1991 “Integral Geometry on Trees", American Journal of Mathematic 113, 441-470.

BERENSTEIN, C. A.

2000 Local tomography and related problems, AMS Contemporary Mathematics, Vol. 278.

COATES, M., HERO IIl, A., NOWAK, R. and YU, B.

2002 "Internet tomography". IEEE Signal processing magazine, may.

CURTIS, E. B., and MORROW, J. A.

1990 Determining the resistors in a network, SIAM J. Appl. Math., Vol. 50, No. 3, pp. 918 930, June.

1990 The Dirichlet to Neumann problem for a resistor network, AMS.

CHUNG, $\mathrm{F}$.

1997 Spectral Graph Theory, AMS.

GAVILÁNEZ, F., BERENSTEIN, C. and BARAS, J.

2004 Continuous and discrete inverse conductivity problems. AMS, Contemporary Math Vol. 362 . 


\section{Franklin Gavilánez}

GAVILÁNEZ, F., BERENSTEIN, C., and BARAS, J.

2006 Network tomography. AMS, Contemporaty Math, Vol. 405.

GAVILÁNEZ, F., BERENSTEIN, C. and BARAS, J.

"Local monitoring of the internet network". Available at http:/techreports.isr.um-

d.edu/ThechReports/ISR/2003/TR/_2003-7/TR\%/_2003-7.phtml

GAVILÁNEZ, F.

2002 "Multiresolution Analysis on the Hyperbolic plane". Master's thesis. University of

GAVILÁNEZ, F. Maryland, College Park.

2006 "Network Tomography", Ph.D. dissertation University of Maryland, College Park. KATSEVICH, A. I. and RAMM, A. G.

1996 The Radon Transform and local tomography. Boca Radon: CRC Press.

MUNZNER, T.

2000 "Interactive Visualization of Large Graphs and Networks", Ph.D. Dissertation, Stanford University, June.

SYLVESTER, and UHLMANN, G.

1987 A global uniqueness theorem for an inverse boundary value problem. Ann. Of Math. (2) 125 , No. 1, 153-169. 